\title{
ANOMALIAS E PATOLOGIAS DENTÁRIAS EM ARCTOCEPHALUS G. SAINT-HILAIRE \& CUVIER (PINNIPEDIA, OTARIIDAE) DA COSTA DO RIO GRANDE DO SUL, BRASIL ${ }^{1}$
}

\author{
César Jaeger Drehmer ${ }^{2}$ \\ Jorge Ferigolo ${ }^{2}$
}

\begin{abstract}
TOOTh ANOMAlies and PATHOlOgies IN ARCtocephalus G. SAINT-HILaire \& CuVIER (PINNIPEdia, OtariIdae) of Rio Grande do Sul Coast, Brazil. The studied species were Arctocephalus australis (Zimmermann, 1783) and Arctocephalus tropicalis (Gray, 1872), (five specimens of each one). Anomalies were discussed under the light of theories which relate the ancestry of pinnipeds with the Ursidae; as well as concerning the evolutionary trends supposed for the Otariidae. Pathologies were related to alimentary and reproductive habits.

KEY WORDS. Pinnipedia, Otariidae, Arctocephalus, anomaly, pathology, teeth
\end{abstract}

Os Pinnipedia Illiger, 1811 recentes apresentam características na dentição que os distinguem totalmente dos carnívoros terrestres (Fissipeda Blumenbach, 1791): ausência total dos carniceiros típicos (primeiro molar inferior e quarto pré-molar superior), dentição decídua reabsorvida intra-uterinamente ou logo após o nascimento (FRECHKOP 1955) e dentição permanente reduzida e simplificada (padrão de cúspides). A semelhança aparente na morfologia dos dentes posteriores ao canino (tendência à homodontia) é expressa no termo "pós-caninos", usualmente sem distinção entre molares e pré-molares (REPENNING et al. 1971), embora os incisivos e caninos sejam bem diferenciados.

Kubota \& Togawa (1964), trabalhando com pinipédios do Hemisfério Norte, e verificando variações na fórmula dentária com relativa freqüência, generalizaram suas conclusões para todo o grupo. Além deste, poucos trabalhos tem se ocupado com a dentição dos pinipédios e mais especificamente com a de Arctocephalus G. Saint-Hilaire \& Cuvier, 1826 (“southern fur seals"). REPENNING et al. (1971) em sua revisão do gênero, referiram o tamanho e a morfologia dos dentes como os caracteres mais diagnósticos para a distinção das oito espécies.

LOUGHLIN (1982) analisou a seqüência na erupção dos pós-caninos em Pinnipedia, incluindo espécimes de Arctocephalus tropicalis (Gray, 1872), comparando-a com a dos Ursidae Gray, 1825 e a dos Mustelidae Swainson, 1835, na determinação de um possível caracter para análise filogenética. CHIASSON (1955) descreveu anomalias em seis crânios da espécie Callorhinus ursinus (Linnaeus,

1) Curso de Pós-graduação em Biociências, Pontifícia Universidade Católica do Rio Grande do Sul.

2) Museu de Ciências Naturais, Fundação Zoobotânica do Rio Grande do Sul. Avenida Salvador França 1427, 90690-000 Porto Alegre, Rio Grande do Sul, Brasil.

Revta bras. Zool. 13 (4): 857 - 865, 1996 
1758). KING (1954) refere a dificuldade no estudo do número de dentes em Arctocephalus porque este é muito variável no caso dos molariformes.

São aqui apresentadas e discutidas anomalias e patologias dentárias nas espécies Arctocephalus australis (Zimmermann, 1783) e A. tropicalis, totalizando cinco espécimes de cada.

\section{MATERIAL E MÉTODOS}

O material utilizado pertence à Coleção de Mastozoologia do Museu de Ciências Naturais da Fundação Zoobotânica do Rio Grande do Sul (MCN-FZBRS), Porto Alegre.

Material examinado. Arctocephalus australis - BRAsilL, Rio Grande do Sul: Palmares do Sul, macho adulto VIII-1992, C. Drehmer leg. (MCN 2497); São José do Norte, fêmea jovem IX-1994, C. Drehmer leg. (MCN 2625); Santa Vitória do Palmar, macho jovem, macho adulto, fêmea adulta IX-1990, J. Ferigolo leg. (MCN 2456, 2461, 2523). Arctocephalus tropicalis - BRASIL, Rio Grande do Sul: Mostardas, macho adulto IX-1994, C. Drehmer leg. (MCN 2620); Santa Vitória do Palmar, três machos adultos, fêmea adulta IX-1994, C. Drehmer leg. (MCN 2605, 2613, 2640, 2609).

Para a preparação, cada espécime foi acondicionado dentro de uma caixa de madeira ou em tanque de fibrocimento, coberto com areia, ficando a céu aberto para que se processasse a decomposição das partes moles. Ao final do período de seis meses a areia foi retirada, peneirada e cada esqueleto foi lavado em água corrente; posteriormente submerso em peróxido de hidrogênio diluído por 24 horas para clareamento.

O dimorfismo sexual no crânio segue o estabelecido por XIMÉNEZ et al. (1984); os termos jovens e adultos estão baseados no nível de fechamento das principais suturas cranianas de acordo com SIMÕES-LoPES et al. (1995).

Abreviaturas utilizadas no texto: $(\mathrm{C} / 1)$ canino inferior; $(\mathrm{PC} / 1-5)$ primeiro a quinto pós-canino inferior; (C1/) caninos superiores; (I3/) terceiro incisivo superior; e (PC1-6/) primeiro a sexto pós-canino superior.

\section{RESULTADOS E DISCUSSÃO}

Fórmula dentária para Arctocephalus: Incisivos 3/2, Caninos 1/1, Pós-caninos 6/5, conforme (REPENNING et al. 1971).

\section{Arctocephalus australis}

MCN 2523 (Fig. 1): somente cinco alvéolos para os pós-caninos superiores, sem nenhuma evidência de fechamento dos alvéolos dos PC6/, o que caracteriza uma redução dentária anômala.

MCN 2497 (Figs 2-3): o PC5/ o o PC/1 esquerdos foram perdidos intra-vitam e os alvéolos fechados, não se podendo, todavia, determinar a causa da perda. A superfície óssea na área de fechamento do alvéolo do PC5/ apresenta-se mais rugosa do que a do alvéolo do $\mathrm{PC} / 1$, o que sugere ter sido este último perdido bem 

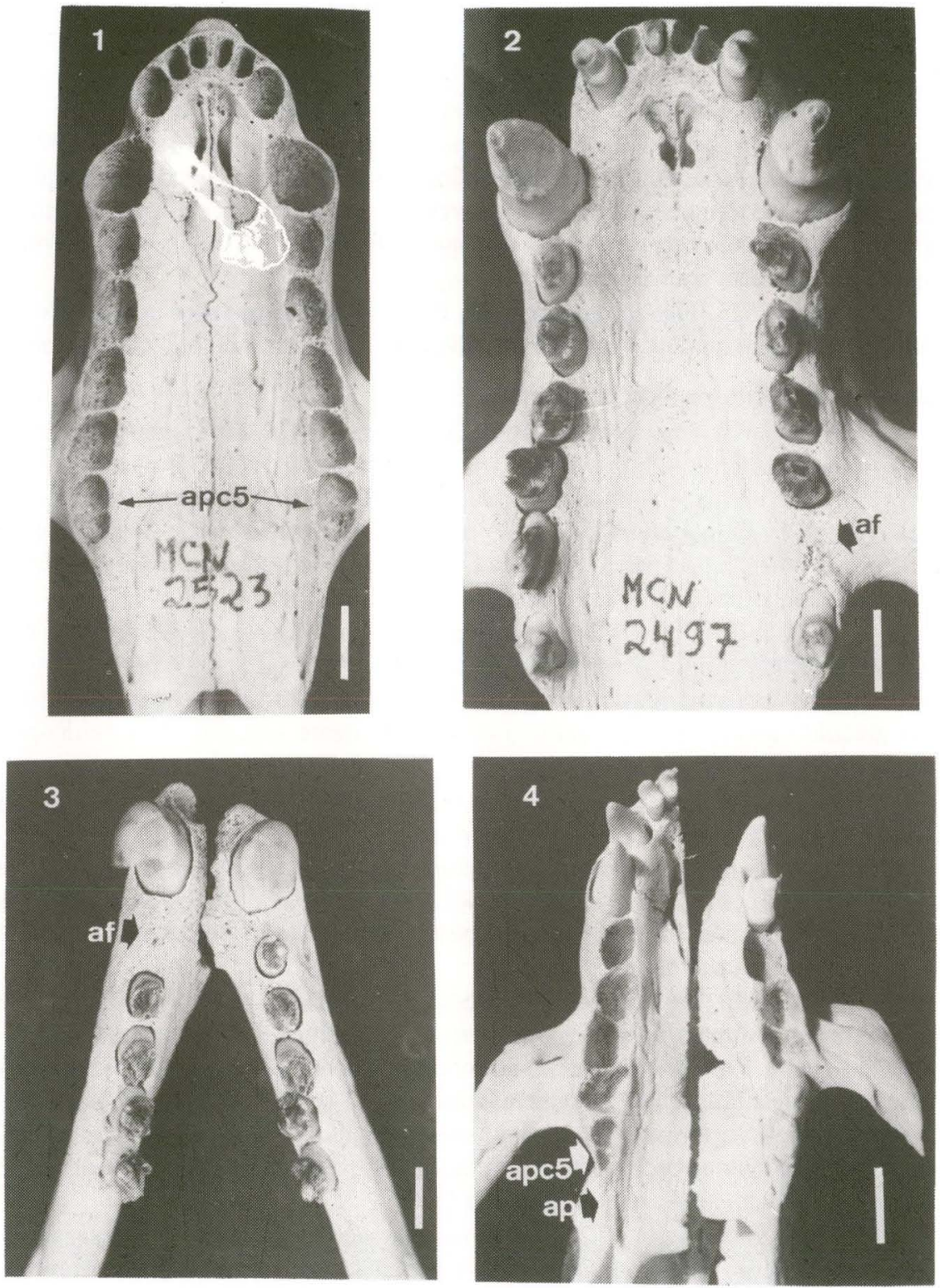

Figs 1-4. Arctocephalus australis. (1-2, 4) Vista palatal parcial (MCN 2523, 2497 e 2456 crânio desarticulado); (3) vista oclusal parcial dos dentários (MCN 2497). (apc5) Alvéolo do pós-canino 5, (af) alvéolo fechado, (ap) apófise pterigóide do maxilar. Barra = $10 \mathrm{~mm}$. 
anteriormente ao primeiro. Estas perdas devem relacionar-se aos hábitos alimentares e a idade do indivíduo (senil), com as suturas cranianas todas já fusionadas.

MCN 2456 (Fig. 4): ausência do alvéolo ou de resquícios deste na posição do PC6/ direito. Em conseqüência disto, a apófise pterigóide do maxilar nesta região é mais longa do que no lado oposto, onde o PC6/ está presente. Sendo um espécime jovem, sem sinal de patologia ou de perda, trata-se de uma redução anômala.

MCN 2461 (Figs 5, 13): dente extra-numerário no maxilar direito, localizado póstero-lingualmente ao $\mathrm{PC} 2 /$ e ântero-lingualmente ao $\mathrm{PC} 3 /$; com raiz dupla e inclinada posteriormente, com um sulco vestibular bem marcado e lingual ausente (Fig. 13). O alvéolo correspondente é menos profundo do que os dos outros dentes, indicando também uma anomalia.

MCN 2625 (Fig. 6): ausência dos PC6/ em ambos os lados (a exemplo do MCN 2523) sem qualquer vestígio dos alvéolos, caracterizando redução anômala.

\section{Arctocephalus tropicalis}

MCN 2609 (Fig. 7): a exemplo do espécime MCN 2497 trata-se de perda intra-vitam, porém do $\mathrm{Cl}$ / esquerdo. O alvéolo está fechado restando na área apenas um sulco bem marcado estendendo-se ântero-posteriormente desde o alvéolo do I3/ até o do $\mathrm{PC} 1 /$. O jugo alveolar do canino mantém-se parcialmente preservado. O $\mathrm{C} / 1$ esquerdo apresenta pouco desgaste e o I/3 está muito fraturado, o que pode estar relacionado a perda do $\mathrm{C1}$ / esquerdo, por provável trauma.

MCN 2605 (Fig. 8): fechamento parcial mas diferenciado dos alvéolos dos $\mathrm{PC} / 2$ esquerdo e direito. $\mathrm{Na}$ área do direito há uma depressão pouco profunda, e na do esquerdo uma algo menos profunda com importante reabsorção na parede lateral do alvéolo, devida provavelmente à oclusão do dente superior. Tais evidências indicam tratar-se de perdas intra-vitam.

MCN 2613 (Fig. 9): ausência do PC6/ direito. O PC5/, o diastema entre o $\mathrm{PC} 4 /$ e o PC5/ e a apófise pterigóide do maxilar no lado direito são maiores que os correspondentes do lado esquerdo. Não há qualquer evidência de que no PC5/ direito haja uma fusão com o PC6/ [semelhante aos dentes geminati de KUBOTA \& TOGAWA (1964)]. Como não há evidência de perda intra-vitam, a ausência do PC6/ é considerada uma anomalia.

MCN 2620 (Figs 10, 14): em ambos os dentários, na mesma posição, ocorre 01 dente extra-numerário localizado ântero-lingualmente ao $\mathrm{PC} / 4$ e póstero-lingualmente ao PC/3. Eles são menores do que os demais, sendo claramente birradiculados com sulcos vestibular e lingual bem marcados (Fig. 14). Tais dentes estão inclinados posteriormente de forma que encostam nos respectivos PC/4. Os alvéolos correspondentes são pouco profundos com reduzidos septos intra-alveolares, bem como os septos inter-alveolares em relação aos $\mathrm{PC} / 3$.

MCN 2640 (Figs 11-12, 15): fechamento quase total dos alvéolos dos PC5/. No lado esquerdo a parte anterior do alvéolo reduziu-se a uma pequena depressão rasa, enquanto que no lado direito apenas a parte posterior do alvéolo assim se apresenta. No dentário esquerdo o PC/5 foi perdido e seu alvéolo fechado, onde se observa uma superfície rugosa, indicando ter sido, possivelmente, perdido em tempo anterior aos outros. Tais perdas foram intra-vitam, provavelmente pós-traumáticas; 

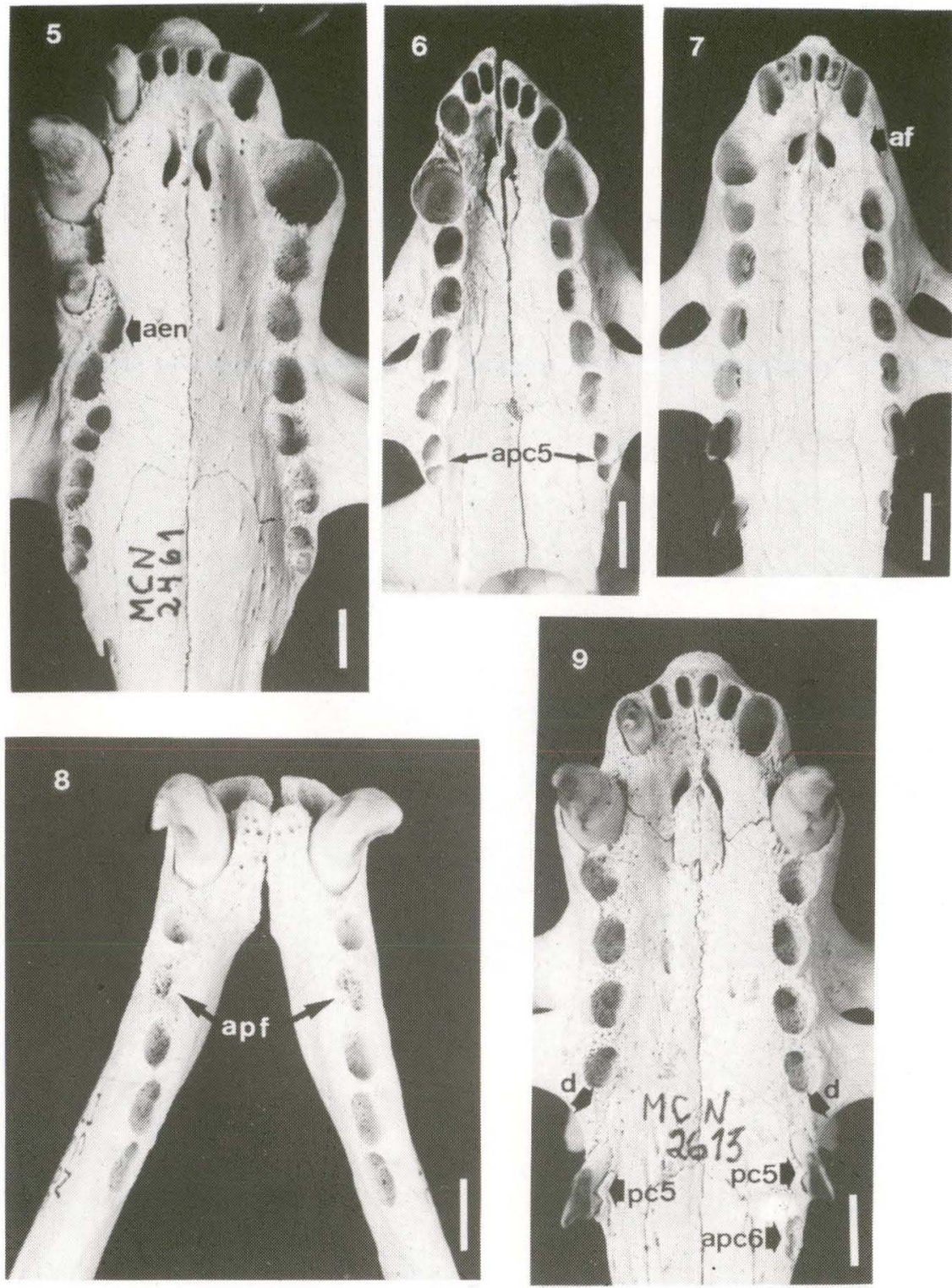

Figs 5-9. (5-6) Vista palatal parcial de Arctocephalus australis (MCN 2461 e 2625); (7-9) Arctocephalus tropicalis. (7, 9) Vista palatal parcial (MCN 2609 e 2613); (8) vista oclusal parcial dos dentários (MCN 2605). (aen) Alvéolo do dente extra-numerário, (af) alvéolo fechado, (apc5) alvéolo do pós-canino 5, (apc6) alvéolo do pós-canino 6, (apf) alvéolo parcialmente fechado, (d) diastema, (pc5) pós-canino 5. Barra $=10 \mathrm{~mm}$. 

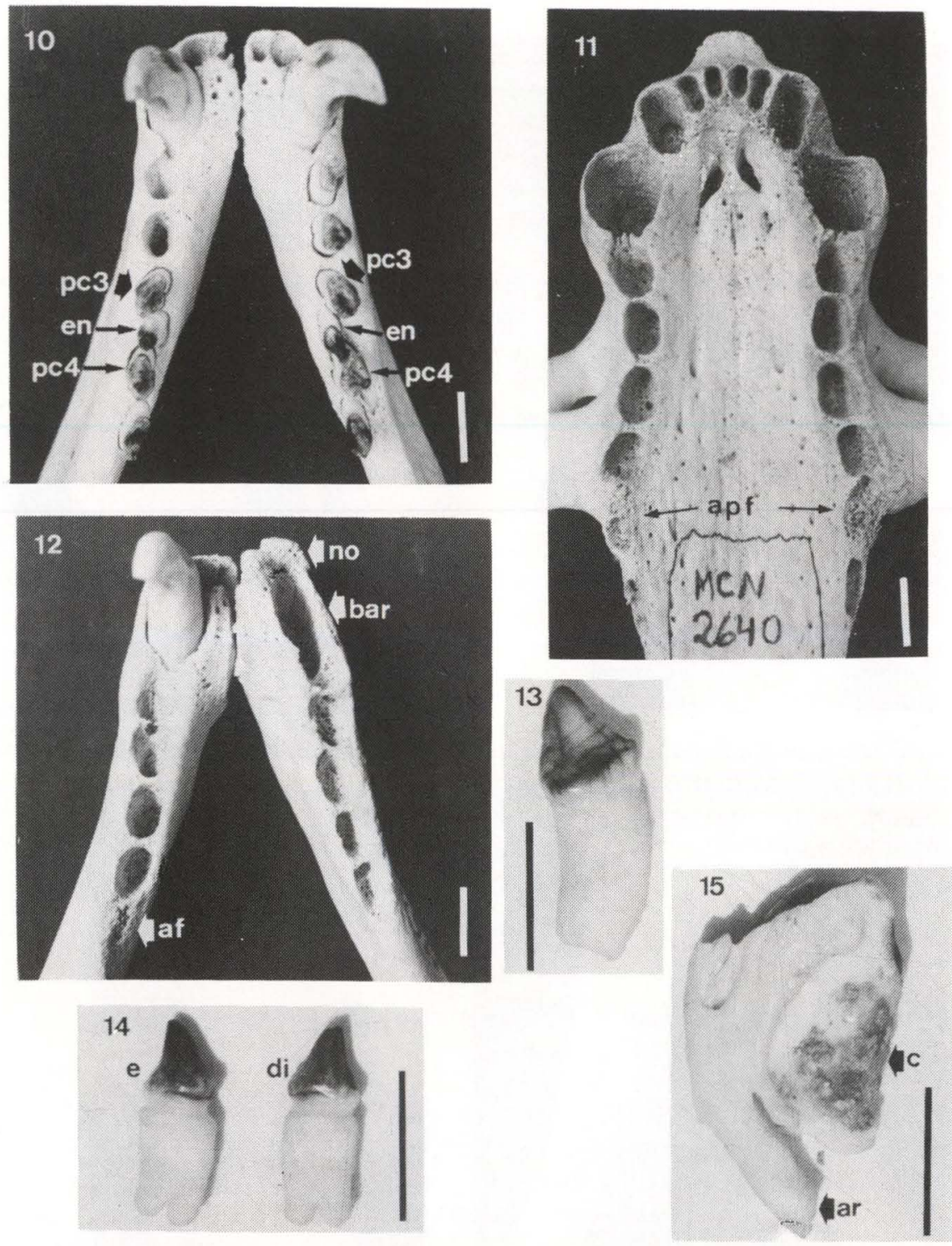

Figs 10-15. (10-12) Arctocephalus tropicalis; $(10,12)$ vista oclusal parcial dos dentários (MCN 2620 e 2640); (11) vista palatal parcial (MCN 2640). (13-14) Vista lingual dos dentes extra-numerários; (13) Arctocephalus australis (MCN 2461); (14) Arctocephalus tropicalis (MCN 2620); (15) vista lingual do canino inferior direito de Arctocephalus tropicalis (MCN 2640). (af) Alvéolo fechado, (apf) alvéolo parcialmente fechado, (ar) ápice da raiz, (bar) borda alveolar reabsorvida, (c) cálculo, (di) dente direito, (e) dente esquerdo, (en) dente extra-numerário, (no) neoformação óssea, (pc3) pós-canino 3, (pc4) pós-canino 4. Barra $=10 \mathrm{~mm}$ ). 
trauma que também se observa, em menor grau, no C/1 direito (Fig. 15), que apresenta múltiplas fraturas na coroa expondo a cavidade pulpar, e ainda periodontite com cálculo, que causou remodelamento do alvéolo, e reabsorção da borda alveolar posteriormente, bem como do cemento e do ápice radicular. Também se observa pequena neoformação óssea na porção anterior do dentário, talvez relacionada à fratura do $\mathrm{C} / 1$. Uma possível lesão peri-apical pode ter ocorrido pois se observa uma pequena solução de continuidade na face medial do dentário, próximo ao alvéolo do PC/1, apesar de não haver indícios de osteomielite.

\section{CONCLUSÕES}

As anomalias descritas revelam a extensão das variações numéricas que podem ocorrer em Arctocephalus, em especial nas duas espécies deste trabalho. Quanto às patologias, principalmente as perdas, não existindo nenhuma evidência de periodontite ou de lesão peri-apical, e havendo evidência da existência anterior do dente (alvéolos fechados), isto permite inferir um trauma, pois se observa grande freqüência de fraturas em graus variados nos dentes em geral, tendo como causa qualquer acidente ou incidente, sendo os mais comuns, pelo que se conhece do comportamento da espécie, defesa de território (no caso de machos), ou acidentes durante a alimentação. Tais fraturas, mais e mais freqüentes nos indivíduos de mais idade devem se relacionar secundariamente ao desgaste do esmalte, ocasião em que os dentes se tornam mais sujeitos a fraturas. A possibilidade de fraturas relacionadas a ingestão de fragmentos de rochas (e.g. BONNER 1981) nos parece pouco provável, pois, além de não ser do comportamento destas espécies (HAMILTON 1934, com relação a $A$. australis), sua possível ingestão não é acidental e portanto não provocaria fraturas dentárias. Há o desgaste natural e constante resultante da oclusão dentária, mais conspícuo nos caninos inferiores, também havendo freqüente desgaste na margem alveolar igualmente resultantes da oclusão dentária. Não foi constatada a presença de cáries em nenhum espécime.

As fraturas em mamíferos são freqüentemente seguidas de complicações, não observadas neste material (a lesão peri-apical do espécime MCN 2640 é apenas uma suspeita). Nas perdas dentárias descarta-se aquelas advindas da coleta e preparação, porque em tais casos os alvéolos estão perfeitamente normais.

Os dentes extra-numerários, que não possuem características de dentes decíduos, devem corresponder a uma reversão a condição primitiva de dentição completa dos carnívoros terrestres, levando-se em conta uma ancestralidade comum com os Ursidae como sugerem BERTA (1991) e WYSS (1987). As reduções dentárias não patológicas corroboram o que BARNES (1989: 11) chama de "... progressive evolutionary loss of the M2 [PC6/] through time in some lineages ... of otariids"; considerando então como caracter mais derivado, para a família Otariidae, uma diminuição no número de dentes pós-caninos. Corroborando tal hipótese, em 4 espécimes há perda dos $\mathrm{PC} 6 /$. Se realmente os dentes dos Otariidae servem pouco para a mastigação (LOUGHLIN 1982), isto significaria que as anomalias (tanto de acréscimo quanto de redução), praticamente não teriam qualquer significado funcional. Tais caracteres corroboram o que se especula a respeito das relações filogené- 
ticas de Arctocephalus com os outros otarídeos; i.e., de que este gênero seria o mais próximo aos otarídeos mais primitivos (e.g. BARNES 1989; MUIZON 1983; REPPENING et al. 1971). A espécie Arctocephalus gazella (Peters, 1785) apresenta o PC5/ e o PC/6 com redução extrema, a ponto de estar praticamente "ausente" a coroa dos dentes (REPENNING et. al. 1971). Neste aspecto esta espécie teria a condição dentária mais derivada, de acordo com a tendência geral referida por BARNES (1989).

AGRADECIMENTOS. A CAPES e ao CNPq, através do seu programa RHAE, que financiaram a execução deste trabalho e a Fundação Zoobotânica do Rio Grande do Sul que deu suporte ao desenvolvimento deste. Ao colega J. L. Maciel pelo auxílio na confecção das fotografias.

\section{REFERÊNCIAS BIBLIOGRÁFICAS}

BARNES, L.G. 1989. A new enaliarctine pinniped from the Astoria Formation, Oregon, and a classification of the Otariidae (Mammalia:Carnivora). Contr. Science, Los Angeles, 403: 1-26.

BERTA, A. 1991. New Enaliarctos* (Pinnipedimorpha) from the Oligocene and Miocene of Oregon and the Role of "Enaliarctids" in Pinniped Phylogeny. Smithson. Contr. Paleob., Washington, 69: 1-33.

BonNER, W.N. 1981. Southern Fur Seals - Arctocephalus (Geoffroy Saint-Hilaire and Cuvier, 1826), p.161-208. In: S.H. RIDGWAY \& R.J. HARRISON (Eds). Handbook of Marine Mammals. The Walrus, Sea Lions, Fur Seals and Sea Otter. New York, Academic Press, vol. I, XIV+235p.

ChIASSON, R.B. 1955. Dental abnormalities of the Alaskan Fur seal. Jour. Mammal., Baltimore, 36 (4): 562-564.

FreChKoP, S. 1955. Ordre des Pinnipèdes, p.292-340. In: P. GrassÉ (Ed.). Traité de Zoologie. Mammifères, Les Ordres: Anatomie, Éthologie, Systématique. Paris, Masson et Cie, tome XVII, fasc. 1, 1170p.

HAMILTON, J.E. 1934 The southern sea lion Otaria byronia (de Blainville). Discovery Rep., Cambridge, 8: 269-318.

KING, J.E. 1954. The otariid seals of the Pacific Coast of America. Bull. Br. Mus. nat. Hist. Zool., London, 2 (10): 309-337.

Kubota, K. \& S. Togawa. 1964. Numerical Variations in the Dentition of some Pinnipeds. Anat. Rec., Philadelphia, 150: 487-502.

LoUGHLIN, T.R. 1982. Functional adaptation of eruption sequence of pinniped postcanine teeth. Jour. Mammal., Baltimore, 63 (3): 523-525.

Muizon, C. DE. 1978. Arctocephalus (Hydrarctos) lomasiensis, subgen. nov. et nov. sp., un nouvel Otariidae du Mio-Pliocene de Sacaco (Perou). Bull. Inst. Fr. Et. And., Paris, 7 (3-4): 169-188.

Repenning, C.A.; R.S. Peterson \& C.L. Hubbs. 1971. Contributions to the systematics of the Southern fur seals, with particular reference to the Juan Fernández and Guadalupe species. Antarctic Res. Ser., Washington, 18: 1-34. SimÕES-LoPES, P.C.; C.J. DREHMER \& P.H. OTT. 1995. Nota sobre os Otariidae e Phocidae (Mammalia, Carnivora) da costa norte do Rio Grande do Sul e Santa 
Catarina, Brasil. Biociências, Porto Alegre, 3 (1): 173-181.

WYSS, A.R. 1987. The walrus auditory region and the monophyly of the pinnipeds. Am. Mus. Novit., New York, 2871: 1-31.

Ximénez, I.; M. Lima; A. Ponce de León; L.M. Batallés \& A. Malek. 1984. Estudio Estadístico de Relaciones Craneales en el "Lobo Fino" Sudamericano, Arctocephalus australis (Zimmermann, 1783). An. Ind. Lobera Pesq. Estado, Montevidéo, 33p.

Recebido em 08.V.1996; aceito em 02.XII.1996. 